\title{
The interplay between stereopsis and structure from motion
}

\author{
MARK NAWROT and RANDOLPH BLAKE \\ Vanderbilt University, Nashville, Tennessee
}

\begin{abstract}
In a series of psychophysical experiments, an adaptation paradigm was employed to study the influence of stereopsis on perception of rotation in an ambiguous kinetic depth (KD) display. Without prior adaptation or stereopsis, a rotating globe undergoes spontaneous reversals in perceived direction of rotation, with successive durations of perceived rotation being random variables. Following $90 \mathrm{sec}$ of viewing a stereoscopic globe undergoing unambiguous rotation, the $\mathrm{KD}$ globe appeared to rotate in a direction opposite that experienced during the stereoscopic adaptation period. This adaptation aftereffect was short-lived, and it occurred only when the adaptation and test figures stimulated the same retinal areas, and only when the adaptation and test figures rotated about the same axis. The aftereffect was just as strong when the test and adaptation figures had different shapes, as long as the adaptation figure contained multiple directions of motion imaged at different retinal disparities. Nonstereoscopic adaptation figures had no effect on the perceived direction of rotation of the ambiguous $\mathrm{KD}$ figure. These results imply that stereopsis and motion strongly interact in the specification of structure from motion, a result that complements earlier work on this problem.
\end{abstract}

This paper documents the interplay between stereopsis and motion information in the generation of threedimensional (3-D) surface perception in human vision. It has long been known that retinal disparity can create a robust sensation of depth and solidity (Wheatstone, 1838), and its effectiveness is most dramatically revealed in the case of texture stereograms devoid of monocular information about shape (Julesz, 1971). Equally impressive is the recovery of shape information when an object is viewed under conditions of motion (Wallach \& O'Connell, 1953). Motion information can even reveal the surface structure of an object that is literally invisible when stationary. This well-known effect, sometimes called the kinetic depth effect (KDE), has received much attention in recent years (Braunstein \& Andersen, 1984; Lappin \& Fuqua, 1983; Sperling, Landy, Dosher, \& Perkins, 1989; Todd, 1984, 1985).

As numerous authors, including Helmholtz (1909/ 1962), have pointed out, stereopsis and motion parallax are comparable geometrically. In the case of stereopsis, the brain utilizes two views of a scene from slightly different perspectives (i.e., the difference in location of the two eyes) to derive a description of an object's depth and shape. Similarly, from a single vantage point, slightly different views of an object over time form the basis for the KDE. In one instance, information is integrated over

Preliminary results from some of these experiments have been reported in Nawrot and Blake, 1989. This work was supported by NIH Grant EY07760 and NIH Vision Core Grant P30-EY08126. We thank Myron Braunstein for helpful discussion. Correspondence should be addressed to Randolph Blake, Department of Psychology, Vanderbilt University, Nashville, TN 37240. space; in the other instance, information is integrated over time. Besides their geometric similarities, stereopsis and motion parallax also yield comparable levels of performance on tasks involving the measurement of depth sensitivity (Rogers \& Graham, 1982). Moreover, both stereopsis and motion parallax yield equivalent variations in perceived depth that are dependent on the retinal orientation of the depth gradient (Rogers \& Graham, 1983).

Stereopsis and motion are also similar in that both are degraded at equiluminance. Lu and Fender (1972) found that stereopsis with random-dot stereograms was markedly impaired when the chromatic stereopair was equiluminant. Similarly, Ramachandran and Gregory (1978) reported that moving contours at equiluminance yield very feeble motion perception. The exact extent to which stereopsis and motion perception are impaired at equiluminance remains unsettled (Shapley, 1990).

Are these similarities between motion parallax and stereopsis coincidental or do they reveal important linkages in the underlying processing architecture? Several lines of evidence suggest that the similarities are more than coincidental. For example, a significant correlation has been reported between an observer's level of competence on a stereopsis task and that person's ability to judge depth when given motion information. Specifically, Richards and Leiberman (1985) tested observers with varying degrees of impairment in stereopsis, as indexed by a deficiency to judge depth from disparity information. Next, Richards and Leiberman measured the ability of these observers to identify and judge the apparent 3-D size of a dynamic 2-D display. The correlation between stereo performance and kinetic depth was $\mathbf{0 . 7 2}$. Richards and Leiberman also observed that people who performed particularly well on 
stereo displays containing crossed disparity information also tended to exhibit better performance on the KD task, whereas stereo performance with uncrossed disparities was less predictive of KD performance. Richards and Leiberman concluded that the outputs from kinetic depth mechanisms and from convergent stereoscopic depth mechanisms are combined prior to an object's assignment to a given depth. While not quarreling with this conclusion, Bradshaw, Frisby, and Mayhew (1987) found that observers were adept at detecting structure from motion in displays imaged entirely at uncrossed disparities; this led them to question the tightness of the linkage between crossed disparities and $\mathrm{KD}$.

Quite recently, Howard and Simpson (1989) reported that the gain of optokinetic nystagmus varies inversely with binocular disparity, which they attributed to neurons sensitive to both disparity and direction of motion. As Richards (1985) pointed out, linkage between stereopsis and motion processing could serve to resolve ambiguities inherent when disparity information or motion parallax information is available on its own.

Cross-adaptation studies also imply some form of interaction in the processing of stereoscopic information and kinetic depth information. Smith (1976) found what he termed a "contingent depth aftereffect" with Lissajous figures (a type of KD display easily generated on an oscilloscope by applying the same signal to the horizontal and vertical amplifiers; see Braunstein, 1962). Viewed normally, a Lissajous figure undergoes spontaneous reversals in the direction of apparent rotation. However, placement of a neutral density (ND) filter in front of one eye produces a strong bias in the perceived direction of rotation, presumably by generating a retinal disparity cue from interocular delay (i.e., the Pulfrich effect). Smith found that when the ND filter was removed following a 30-sec observation period of unambiguous motion, the Lissajous figure appeared to rotate in the direction opposite that experienced with the filter in place. The stereo cue, in other words, disambiguated the motion cue, and this disambiguation in turn yielded a pronounced visual aftereffect. A rather similar cross-adaptation result was described by Rogers and Graham (1984). They had observers stare at a corrugated surface whose depth undulations were unambiguously defined either by binocular disparity or by motion parallax created by perspective projection of motion translations. ${ }^{1}$ Following this period of adaptation to depth, the observers viewed an ambiguous surface whose periodic depth corrugations could be seen in either of two phases. The observers reported that the perceived peaks and troughs appeared spatially in antiphase to the actual undulations experienced during adaptation. This aftereffect was measurable even when the unambiguous adaptation surface was specified by motion parallax and the ambiguous, postadaptation surface was specified by stereopsis, and vice versa.

Contrary to the above results, however, Wallach, Moore, and Davidson (1963) found that KD was unaffected by prior adaptation to stereoscopic depth. In their study, Wallach et al. used a mirror stereoscope to exaggerate the retinal disparities associated with viewing a rotating wire figure. Following prolonged inspection of this exaggerated stereoscopic depth, observers perceived a stationary version of the figure to be flattened, or compressed in depth, even though it contained the same exaggerated disparities. This so-called satiation effect was not observed, however, when the test figure was a monocularly viewed $\mathrm{KD}$ version of the figure, implying that stereoscopic satiation did not generalize to motion. In a later paper, Wallach and Karsh (1963) proposed that the initial effect of adaptation to exaggerated disparity results from the discrepancy between the depth signaled by KD and the depth signaled by the exaggerated disparities. According to this view, then, kinetic depth information plays an essential role in the modification of stereoscopic depth perception.

In the present paper, we give the results of a series of psychophysical experiments in which we examined in closer detail the interactions between stereopsis and motion information in the specification of structure from motion. We utilized a version of the cross-adaptation paradigm, whereby adaptation to a stereoscopically defined, rotating object subsequently biases the perception of rotation of an object defined solely by motion information. In particular, we have assessed the necessary and sufficient stimulus conditions for adaptation by various 3-D figures to affect the perception of subsequently presented 2-D figures.

\section{METHOD}

\section{Stimuli and Displays}

The stimuli for these experiments consisted of computer-generated random-dot cinematograms depicting objects rotating in depth. Each frame in a given cinematogram was a 2-D, parallel projection "snapshot" of the object at some degree of rotation around a stationary axis in the mathematically defined 3-D coordinate system. Presenting the cinematogram frames quickly in succession on the face of the video monitor produced the KDE of a rotating object. Although the $\mathrm{KD}$ figure presented on a single monitor appears vividly to be 3-D, it is devoid of retinal disparity information and is, hence, nonstereoscopic. Therefore, we will term the KD figure without disparity "2-D" and the KD figure with disparity information " $3-\mathrm{D}$." It is important to keep in mind that the terms 2-D and 3-D refer to the absence or presence of disparity information, not to the 3-D quality of the resulting percept.

With these random-dot cinematograms, stereoscopic depth is generated by presenting separate views of the 2-D figure to the left and right eyes; slight differences between each eye's view simulate the conditions experienced with natural viewing. In a given instance, one eye views a slightly earlier or later frame in the cinematogram sequence than does the other eye (Dosher, Sperling, \& Wurst, 1986). The resulting stereoscopic information unambiguously specifies the direction of the figure's rotation; accordingly, we will refer to this as the 3-D display, in contrast with the ambiguous 2-D display.

The basic stimulus comprised 200 small $\left(2^{\prime} \times 2^{\prime}\right)$ black dots depicting random placement on the surface of a 3-D sphere with a diameter of $2.5^{\circ}$. The cinematograms were created by rotating the sphere $2^{\circ}$ about its vertical axis between the generation of each frame, thereby using 180 frames to depict the entire revolution of 
the sphere. The cinematograms were shown with a $30-\mathrm{msec}$ frame rate. To promote stable fixation during stereoscopic adaptation, a small cross $\left(6^{\prime} \times 6^{\prime}\right)$ was shown in the center of the cinematograms. A larger cross extended from the top and sides of the 180-pixel $\left(3^{\circ}\right)$ diameter aperture (within which the cinematogram was presented) to the edges of the monitor. This larger cross helped keep the fixation plane in register. An illustration of this stimulus configuration appears in Figure 1.

\section{Apparatus}

The cinematograms were generated and presented with a Macintosh II computer on two matched ${ }^{2}$ monochrome monitors $(66.7-\mathrm{Hz}$ noninterlaced frame rate; $\mathrm{P} 4$ phosphor; 72 pixels/in.). While comfortably seated in a darkened room, the observer viewed these matched monitors through a mirror stereoscope from an effective viewing distance of $114 \mathrm{~cm}$. The observer used the keys on the computer terminal to signal responses, which were recorded by the computer.

\section{Procedure}

Each trial consisted of a 90 -sec adaptation period immediately followed by a 15-sec test period. Throughout the entire adaptation/test sequence, the observer tracked the direction of rotation of the figure by pressing one key to signal clockwise rotation (viewed from the top) and another key to signal counterclockwise rotation. The direction of perceived rotation was tracked throughout the entire adaptation and test portions of a trial, and following each trial the observer rested for at least 2 min.

Observers completed a set of four trials with clockwise adaptation and a set of four trials with counterclockwise adaptation; the order of these two sets of trials was random. At least a 5-min break was interposed between the two sets of trials, and an eight-trial session was usually completed within an hour.

In earlier experiments (e.g., Rogers \& Graham, 1984), observers simply indicated the appearance of the test display immediately following adaptation. However, we had observers track the test display's direction of rotation for a period of time after adaptation. This tracking measure provides a more complete index of the effectiveness of adaptation and information on the time course of recovery from adaptation.

\section{Observers}

The observers consisted of 2 naive, paid observers-one with normal visual acuity (L.G.) and one with visual acuity corrected to normal (D.T.)- and the 2 authors (M.N. and R.B.), both with normal visual acuity. A few additional people within our laboratory (all naive) also participated in the first experiment.

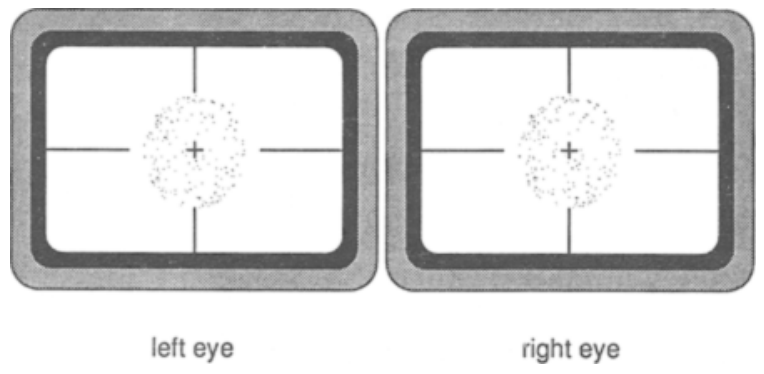

Figure 1. Schematic of left- and right-eye displays (not drawn to scale). Cinematograms could be presented separately to the two eyes, and except where noted, the rotating figure appeared centered on the display. A fixation mark and set of cross-hairs served as binocular fixation stimuli and defined the plane of zero disparity. The globe figure shown here represents one pair of stereoscopic frames from a cinematogram depicting a 3-D rotating globe.

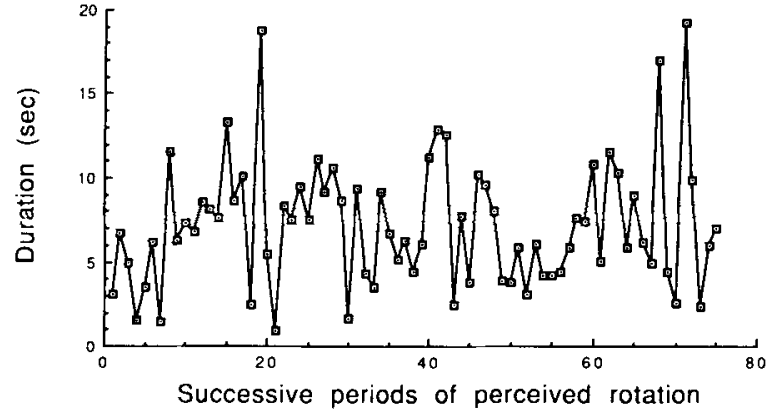

Figure 2. Successive durations of perceived rotation of an ambiguous 2-D globe. Individual durations fluctuate randomly over time. A regression line fit to these data (not shown) had a negligible slope, indicating no tendency for durations to increase over the 10-min tracking period.

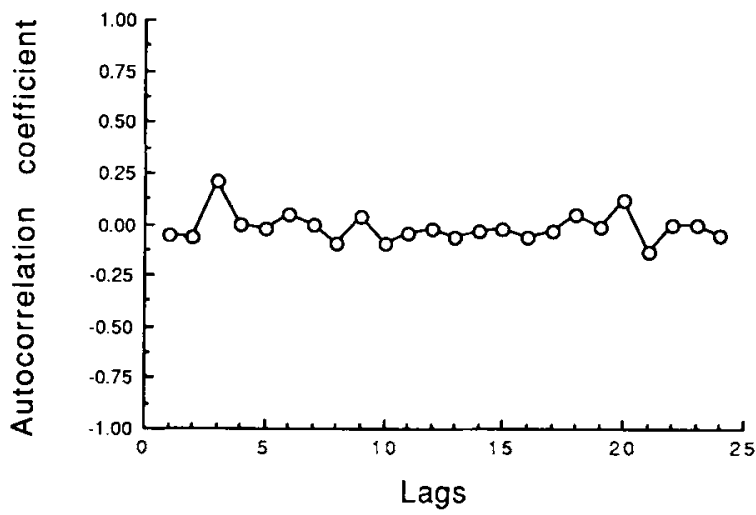

Figure 3. Autocorrelation coefficients over 25 lags. This analysis was performed on the durations depicted in the previous figure.

\section{RESULTS}

\section{The Basic Phenomenon}

When viewing the orthogonal projection of a rotating $\mathrm{KD}$ figure, an observer experiences spontaneous reversals $^{3}$ in the perceived direction of rotation (Fisichelli, 1947; Howard, 1961). We measured the temporal properties of these spontaneous perceptual reversals by having an observer track the reversals of a rotating 2-D globe (i.e., identical cinematograms presented to the two eyes) for $10 \mathrm{~min}$. The results from this experiment are shown in Figure 2. There is no obvious periodicity in the successive durations of perceived rotation, nor is there a tendency for durations to lengthen over time. To test specifically for stochastic independence, an autocorrelation analysis was performed on the successive durations, for 25 lags. The resulting correlation coefficients, shown in Figure 3, were small and fluctuated irregularly around zero; this analysis hence reveals no hint of temporal dependencies in the successive durations of perceived rotation. Figure 4 shows the frequency distribution of these individual durations. 


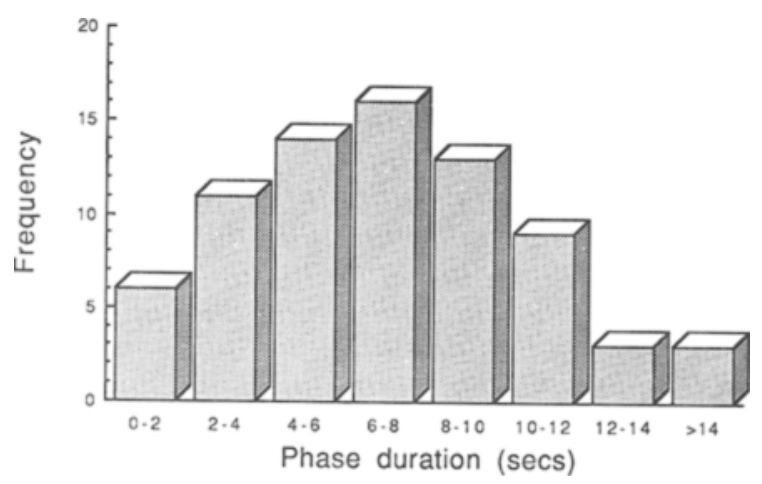

Figure 4. Frequency distribution of the individual durations of perceived rotation depicted in Figure 2.

In summary, individual durations of perceived rotation in a given direction resemble the durations of perception found with other kinds of ambiguous figures (Borsellino, De Marco, Allazetta, Rinesi, \& Bartolini, 1972; De Marco et al., 1977) as well as the durations of binocular rivalry phases (Fox \& Herrmann, 1967).

\section{Disambiguating the Perceived Direction of Rotation}

Retinal disparity information effectively disambiguates the direction of rotation of a KD figure (see, e.g., Dosher et al., 1986). In particular, when a given cinematogram frame is presented to the left eye slightly in advance of its presentation to the right eye, the object appears to rotate clockwise (Burr \& Ross, 1979). Reversing the temporal order (i.e., right eye leading the left eye) causes the perceived direction of rotation to be counterclockwise. For most observers with normal stereo vision, this retinal disparity information stabilizes the perceived direction of rotation.

\section{Adaptation of 2-D Motion by Stereopsis}

For our basic experiment, we had observers adapt to a rotating 3-D (i.e., stereoscopic) globe and then track fluctuations in the perceived direction of rotation of an ambiguous 2-D globe. After adaptation to the stable 3-D figure, the rotation of the previously ambiguous 2-D figure was predominantly perceived to be in the direction opposite that experienced during the adaptation period (see Figure 5).

Recovery from adaptation. Casual observation suggested that $90 \mathrm{sec}$ of stereoscopic adaptation effectively biased the 2-D figure's direction of rotation for somewhat less than a minute. To measure the actual time course of recovery from 3-D adaptation, we had 2 observers track the perceived direction of rotation of the 2-D globe for $75 \mathrm{sec}$ following a 90 -sec period of stereoscopic adaptation. Figure 6A divides this 75 -sec postadaptation period into 15-sec bins and shows for each bin the proportion of time the observer perceived the 2-D globe to be rotating in a direction opposite that present during adaptation.
Recovery seems to be complete (i.e., either direction is equally probable) within $45 \mathrm{sec}$.

In a second experiment, we interspersed a variableduration "blank" period between the 90 -sec adaptation phase and a 15-sec test phase. During this blank period, the observer kept the eyes closed until an auditory signal cued the observer to open the eyes and track the rotation of the 2-D globe. Figure $6 \mathrm{~B}$ shows the total time during the 15-sec test period that the observer experienced the 2-D globe to be rotating in a direction opposite that seen during adaptation. Here it can be seen that the aftereffect decays monotonically during this blank period, although the rate of decay is less than that measured when motion is experienced throughout the period following adaptation (i.e., compare the slopes of the curves in Figures 6A and 6B).

Retinal specificity of the aftereffect. Earlier experiments had yielded evidence for perceptual coupling between the perceived direction of rotation of two spatially separated 2-D rotating objects (Eby, Loomis, \& Solomon, 1989). Such KD figures, in other words, appear to rotate in the same direction, and they reverse directions in synchrony. This coupling suggests that the neural events underlying $K D$ are rather global in nature. Is the effect of 3-D adaptation on 2-D motion also spatially extensive? To answer this question, we made measurements with the figure viewed during adaptation imaged on a different area of the retina from that on which the test figure was imaged.

For this experiment, the center of the 3-D adaptation globe was situated $1^{\circ}$ to the left of the fixation mark, while the 2-D test figure was imaged $1^{\circ}$ to the right of fixation.

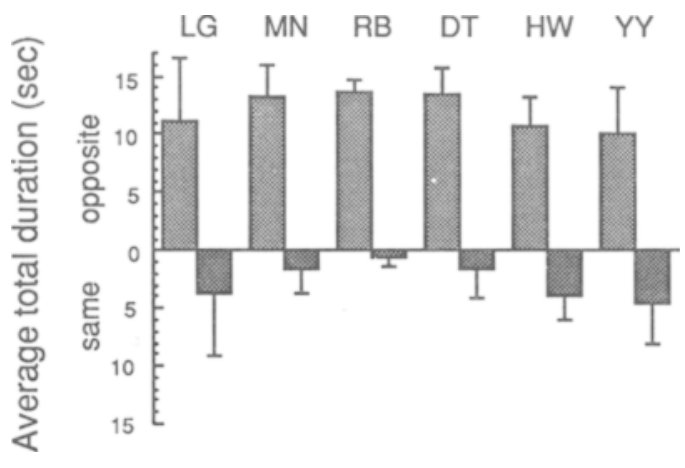

Figure 5. Effect of $90 \mathrm{sec}$ of adaptation to a rotating 3-D (stereoscopic) globe on perceived direction of rotation of an ambiguous 2-D globe viewed for a 15-sec test period immediately following adaptation. Each histogram is the average of eight adaptation/ test episodes, four with the adaptation globe rotating clockwise and four with it rotating counterclockwise. Values plotted below the zero line indicate rotation in the direction experienced during adaptation (same) and values above this line indicate rotation in the direction opposite that experienced during adaptation (opposite). Vertical bars denote $1 S D$. The test figure showed a pronounced tendency to be seen rotating in the direction opposite that experienced during adaptation, regardless of which adaptation direction was experienced. 
A
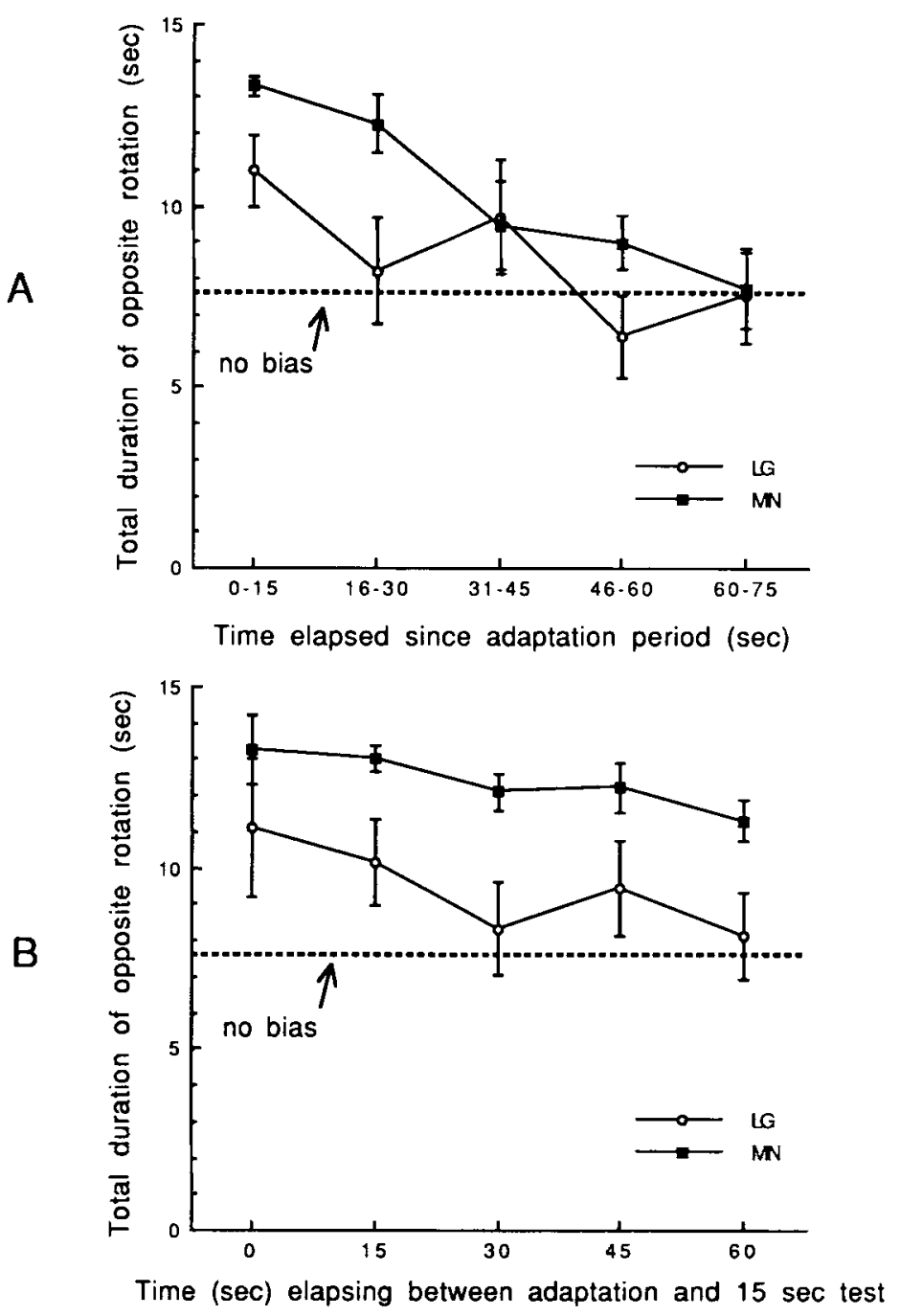

Figure 6. Recovery from 3-D adaptation. Each data point gives the average $(n=4)$ total duration that the 2-D test figure appeared to rotate in a direction opposite that experienced during an initial 90-sec period of 3-D adaptation. In both panels, a value of 7.5 sec indicates no biasing effect. The vertical bars represent $1 S E$. (A) Each data point summarizes the results for successive 15sec periods following the end of adaptation. (B) Each data point summarizes the results for a 15-sec test period administered sometime after the termination of adaptation, with the abscissa specifying the duration elapsing between the end of adaptation and the beginning of the test.

The observers were instructed to maintain fixation on the center cross at all times (fixation was not objectively monitored). Two observers were tested under these conditions, with four trials devoted to each of two adapting directions of rotation. Adaptation lasted $90 \mathrm{sec}$ and the 15 -sec test period followed immediately. As summarized in Figure 7A, both directions of rotation were experienced following 3-D adaptation. This failure of adaptation to bias perceived rotation implies that the adaptation effect is retinally specific, not global. The same pattern of results was found when the adaptation figure appeared $1^{\circ}$ above the fixation mark and the test figure appeared $1^{\circ}$ below fixa- tion. The latter finding implies that the results in Figure 7A are not attributable to weak interhemispheric connections.

To be certain that peripheral stimulation per se was not responsible for these results, a control condition was included, in which the adaptation and test figures both appeared $1^{\circ}$ to the left of fixation. Now, with both figures imaged on the same retinal area, adaptation was very effective in temporarily disambiguating the perceived direction of rotation, as is shown in Figure 7B. Thus peripheral stimulation alone is not responsible for the results in Figure 7A. 


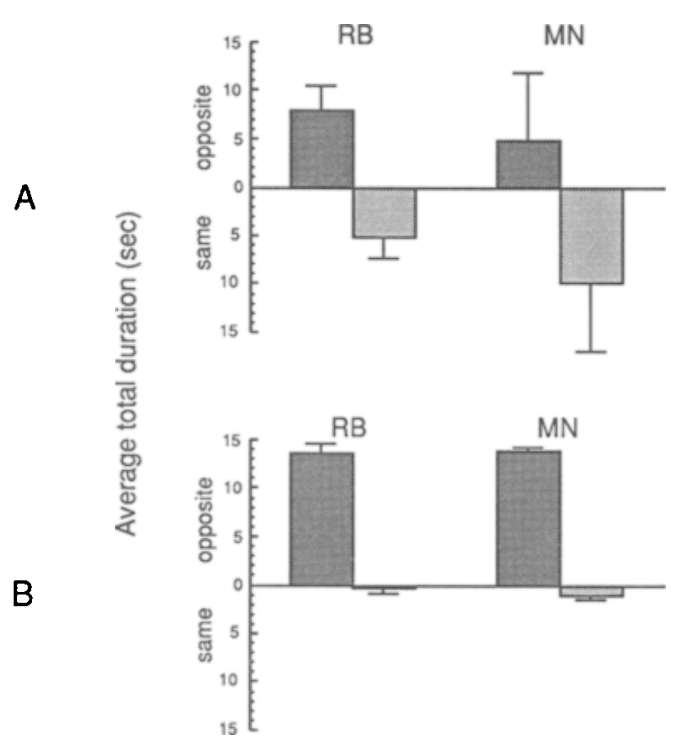

Figure 7. Average ( $n=8$; vertical bars denote $1 S D$ ) total duration of perceived rotation in the direction experienced during adaptation (same) and in the direction opposite that experienced during adaptation (opposite). (A) 3-D adaptation figure and 2-D test figure were imaged on different retinal areas; adaptation had no effect. (B) Adaptation and test figures were imaged on the same retinal areas, parafoveally from the fixation mark; adaptation had a pronounced effect.

Next we tried imaging the adaptation and test figures on more nearly adjacent retinal areas, to learn whether the spread of adaptation might be found over smaller retinal distances. To implement this experiment, we halved the diameter of the adaptation and test globes, so that the 100 dots comprising the figure were contained within a $1.25^{\circ}$ diameter circular region. When imaged on the same retinal area, the small 3-D adaptation globe was very effective in biasing perception of the small 2-D test globe. When, however, the 3-D adaptation globe was situated up and to the left of fixation and the 2-D test figure was placed down and to the left, no biasing effect on perceived rotation was found. This was true even though the two small figures together occupied an area of the retina no larger than that stimulated by a single, normally sized globe.

Finally, we tried a condition in which the 3-D adaptation and 2-D test figures appeared in the same location in visual space but were imaged on different retinal areas during adaptation and during test. For this condition, the adaptation and test figures both appeared exactly in the center of the display (i.e., objectively straight ahead from the observer's vantage point). During adaptation, the observer fixated the left-hand edge of the 3-D figure, and during testing, the observer fixated the right-hand edge of the 2-D figure. (Thus the two rotating figures appeared in the same visual location, although they were imaged on different retinal areas.) This condition produced no bias in the perceived direction of rotation of the test figure.

These observations thus lead us to conclude that stereoscopic adaptation is tied to retinal coordinates, with very little spatial spreading. Evidently the mechanisms underlying adaptation differ from those responsible for perceptual linkage between multiple KD figures (Eby et al., 1989). In the same vein, Toppino and Long (1987) found that the reversals in the perceived direction of a rotating Necker cube could be influenced by local, but not global, adaptation.

Adaptation to flat planes of motion seen in depth. Petersik, Shepard, and Malsch (1984) have proposed the existence of mechanisms responsive to the direction of rotation of objects in 3-D space. Such putative mechanisms could be invoked to explain the results reported in this paper. Rather than the "direction-selective rotation detectors" of Petersik et al., however, one could also envision a simpler explanation in which adaptation takes place within unidirectional motion sensors (e.g., mechanisms of the sort posited by Watson \& Ahumada, 1985) that are disparity-selective. These mechanisms would be selectively stimulated by the front and rear surfaces of a rotating figure. To test the feasibility of this simpler explanation, we performed the following experiment.

We created a stimulus that presumably would adapt mechanisms specific for motion and stereo depth, but not mechanisms responsive to rotation specifically. The display consisted of two sheets of dots moving in opposite directions, with one sheet placed in crossed disparity relative to the plane of fixation and the other sheet placed in uncrossed disparity. Each plane was composed of 100 dots, and each dot made a horizontal excursion of 2 pixels from frame to frame. A new frame was presented every $30 \mathrm{msec}$. The magnitude of the disparities was $2^{\prime}$, an amount that placed the two sheets of dots at the same distance from fixation as the front and rear surfaces of the 3-D globe used in our earlier experiments. The two planes of dots appeared within a circular aperture, $2.5^{\circ}$ in diameter (i.e., the same size as the cross section of the sphere). The observers adapted to this modified 3-D stimulus for $90 \mathrm{sec}$ and then tracked reversals in the direction of rotation of the 2-D sphere.

Adaptation to these multiple planes of moving dots produced a robust aftereffect. When the front plane of dots moved leftward and the back plane rightward, the 2-D globe subsequently appeared to rotate counterclockwise during the 15-sec test period. Reversing the directions of motion of the 3-D planes of dots reversed the biasing effect on the 2-D globe. We next tried adapting observers to a single sheet of moving dots imaged either at $2^{\prime}$ crossed disparity or at $2^{\prime}$ uncrossed disparity (i.e., a single component of the multiplane stereo figure). This condition of adaptation produced no effect on the reversals in direction of the subsequently viewed 2-D figure.

These results are consistent with the hypothesis that adaptation affects direction-selective motion sensors whose activity is disparity-specific. Of course, one can argue that two sheets of dots imaged at different disparities approximate the front and rear surfaces of a large sphere viewed through an aperture. Hence the neural process revealed by 3-D adaptation could still be one designed to signal the direction of 3-D rotation. This argument also 
implies that the conventional motion aftereffect resulting from adaptation to a single sheet of dots flowing in a single direction arises from prolonged stimulation of this putative rotation-signaling mechanism when it "views" just the front surface of a large, opaque sphere exposed through a small aperture. Recall, however, that a single sheet of adapting dots did not bias the direction of rotation of a 2-D figure, which undermines the argument for the existence of a rotation-signaling mechanism. Rather, we favor the idea that 3-D adaptation arises from simultaneous stimulation of direction-selective sensors responsive to different disparities.

Monocular versus Binocular Viewing. In the experiments described so far, the 2-D test figure was presented to both eyes with zero disparity. Would the binocularly viewed 3-D adaptation figure subsequently influence a monocularly viewed 2-D test figure? If this is genuinely a motion-specific disparity aftereffect, it should be possible for a 3-D figure containing disparity to affect the appearance of a 2-D figure viewed monocularly, if monocular viewing is signaled as zero disparity. Can this prediction be realized?

To find out, observers were adapted to the binocularly viewed 3-D sphere for $90 \mathrm{sec}$. Following adaptation, a 2-D sphere was presented to only one eye, with the tested eye varied over trials. The nontested eye viewed only the fixation mark and the fusion cross-hair lines. Observers were instructed, as always, to track the direction of rotation of the 2-D test stimulus. As can be seen in Figure 8, 3 -D adaptation yielded a strong bias in the perceived direction of rotation of the monocularly viewed 2-D target.

How do we explain this finding? Assume that monocular viewing stimulates those neural elements that are responsive to zero disparity under binocular viewing. Prolonged binocular viewing of a stimulus imaged at nonzero disparities produces a temporary shift in the balance of activity associated with viewing a zero-disparity stimulus (including a monocularly viewed target); disparity aftereffects of this sort have been described by Blakemore and Julesz (1971). Our present results simply add the contingency of motion to this formulation. An elaborated ver-

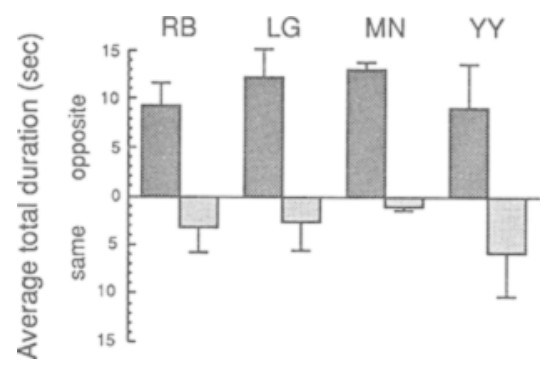

Figure 8. Average $(n=8)$ duration of perceived rotation in the direction experienced during adaptation (same) and in the direction opposite that experienced during adaptation (opposite). The adaptation stimulus was the 3-D rotating globe and the test stimulus the 2-D globe presented to just one eye; the untested eye viewed a blank screen with the fixation mark and cross-hairs. sion of this model has been detailed elsewhere (Nawrot \& Blake, 1990, in press).

Specificity of rotational axis. If 3-D adaptation arises from simultaneous stimulation of direction-selective sensors responsive to a given disparity, adaptation and test figures would need to have common directions of motion for biasing to occur; adaptation and testing with perpendicular axes of rotation should be ineffective. Do orthogonally rotating figures indeed fail to interact?

In one condition in this experiment, the 3-D globe rotated about the horizontal axis while the 2-D globe rotated about the vertical axis; in another condition, the adaptation axis was vertical and the test axis was horizontal. Creation of a stereo cinematogram of the sphere rotating about the horizontal axis required a somewhat different animation procedure from the one used for stereoscopic rotation about the vertical axis. For this cinematogram, two separate films were made of the sphere rotating about the horizontal axis. After one such animation sequence had been generated, a second sequence was made of the same globe shifted $2^{\circ}$ about the vertical axis. Using these two film pairs, the stereoscopically defined direction of rotation about the horizontal axis depended on which eye viewed which film. With one viewing condition, the front of the sphere appeared to move downward, but switching the films viewed by each eye reversed the direction of rotation such that the front of the sphere moved upward.

For each of the two conditions described above, four adaptation/test trials were devoted to each of two directions of rotation (i.e., upward/downward, leftward/rightward). The duration of adaptation was $90 \mathrm{sec}$, and the test period lasted 15 -sec. For none of the 3 observers tested did the 3-D adaptation figure bias the perceived direction of rotation of the 2-D figure. The adaptation and test figures must share directions of motion for one to bias the other.

3-D motion adaptation and a stationary 3-D test figure. The adaptation effect studied in our experiments involves a bias in the perceived direction of rotation of a 2-D figure whose constituent features (i.e., random dots) are actually moving during the test period. This aftereffect should be distinguished from the conventional motion aftereffect, in which a stationary 2-D figure appears to move in a direction opposite that viewed during adaptation to motion-in other words, illusory motion is seen where none actually exists. Now, suppose an observer adapts to a 3-D sphere rotating in one direction and then views a stationary 3-D sphere. Will the stationary sphere appear to rotate in the opposite direction? Such an outcome seems feasible, since it is possible to generate a disparity-specific motion aftereffect (Anstis \& Harris, 1974). To find out whether a rotating 3-D sphere can induce motion in a stationary 3-D sphere, the following twopart experiment was performed.

To begin, the observers adapted to a single sheet of 50 dots moving either leftward or rightward within a circular aperture $2.5^{\circ}$ in diameter. After the adaptation period, the observers verbally described the appearance of a stationary 3-D globe (i.e., a single pair of cinematogram 
frames from the stereoscopic animation sequence). Three adaptation conditions were tested: (1) the moving adaptation dots were imaged with crossed disparity at the same depth plane as that of the front of the 3-D test sphere; (2) the adaptation dots were imaged with zero disparity at a plane cutting through the middle of the 3-D test sphere; and (3) the adaptation dots were imaged with uncrossed disparity at a plane parallel to the rear surface of the 3-D test sphere. In all three conditions, observers reported a weak motion aftereffect: for a brief time immediately following adaptation, the entire test sphere appeared to drift laterally, in a direction opposite that experienced during adaptation. However, there was absolutely no sense of rotation of the stationary globe, only lateral displacement.

In a set of control measurements, we employed a stationary test figure that was strictly 2-D (i.e., a flat sheet of dots imaged with zero disparity). Following adaptation to motion in one direction at zero disparity, a strong motion aftereffect was elicited: the static dots appeared to move for many seconds in a direction opposite that experienced during adaptation. This, of course, constitutes a simple motion aftereffect.

So in comparison with these control measurements, the motion aftereffect was weakened considerably when the flat sheets of dots seen by the two eyes were stereoscopically fused into a static 3-D sphere. This observation is reminiscent of findings of Fox, Patterson, and Lehmkuhle (1982), who found that the strength of the motion aftereffect created using cyclopean stimuli varied with the similarity between test and adaptation disparities.

In the experiment just described, a single sheet of dots imaged at a given disparity failed to induce apparent rotation in a stationary 3-D figure. In this next experiment, we utilized a rotating stereoscopic sphere for adaptation, not just a single sheet of dots, reasoning that the 3-D sphere might be a more potent figure for inducing apparent rotation in a stationary 3-D sphere. This expectation was not fulfilled, however; the stationary 3-D globe showed no hint of rotation or lateral motion following $90 \mathrm{sec}$ of adaptation to the rotating 3-D globe. Evidently the perceptual bias under study here is not simply a disparitycontingent motion aftereffect. Rather, it appears to be a complex, motion-contingent disparity aftereffect, in that motion is a necessary but not sufficient condition for the occurrence of the aftereffect.

Shape specificity. So far we have concentrated on direction of rotation and disparity selectivity as determinants of this interesting aftereffect, and we have utilized a globe-shaped figure to assess these effects. To what extent is the interaction between stereopsis and KD shapespecific? Imagine, for instance, adapting to a 3-D globe rotating, say, counterclockwise, and then inspecting a 2-D cube whose direction of rotation is ambiguous. Can the globe influence the cube?

To answer this question, we generated two new figures: a cube and a random wire figure. The cube was made by randomly placing 200 dots on the surface of a cube
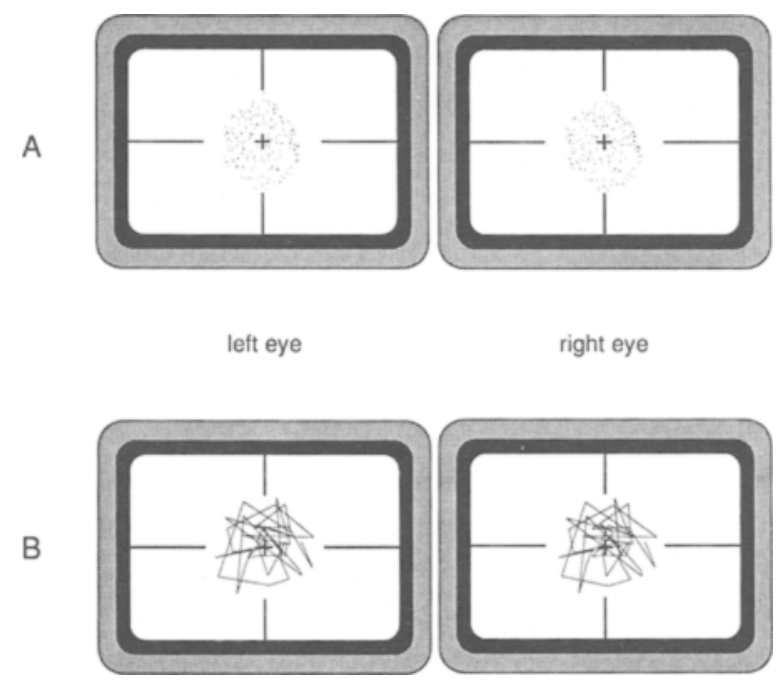

Figure 9. Schematic of cube (panel A) and wire (panel B) figures used to examine the extent to which adaptation and test figures must be identical in shape. Readers capable of free fusion can experience the 3-D shapes of these figures but not, of course, their rotations during movement.

the dimensions of which were 80 min per side. This cube appeared tilted $45^{\circ}$ about its $x$ - and $z$-axes, so that its new vertical axis intersected two opposing corners; a single frame of this stimulus appears in Figure 9A. Cinematograms of the cube were made by rotating the cube $2^{\circ}$ about this vertical axis between each cinematogram frame (i.e., by an amount identical to that used for globe rotation). The random wire figure was made by connecting 41 randomly selected points lying within the volume of the cube. This produced a wire figure with $\mathbf{4 0}$ random but connected 2 ' wide line segments, one example of which is shown in Figure 9B. This object was also rotated $2^{\circ}$ between cinematogram frames, to create the illusion of rotation when the frames were presented in close succession. Using these new stimuli, we performed several experiments.

For one experiment, the observers adapted to a stereoscopic 3-D version of the cube. Following the 90-sec adaptation period, the observers tracked the rotation of a 2-D ambiguous sphere. Four trials were conducted in each of the two biasing directions (clockwise and counterclockwise). As is shown in Figure 10A, the 3-D cube was effective in biasing the direction of rotation of the 2-D globe. Next, we simply reversed the displays, so that the 3-D globe was the adaptation figure and the 2-D cube was the test figure. Again, the biasing effect was potent. Finally, we replaced the cube with the wire figure and repeated these measurements. Again, there was strong interaction between the globe and the wire figure (see Figure 10B).

These results show that figural differences have no influence on the interaction between stereopsis and $\mathrm{KD}$. Of course, this definition of "figural difference" focuses on the global shapes of the adaptation and test objects. It is possible that the globe, cube, and wire figure are com- 


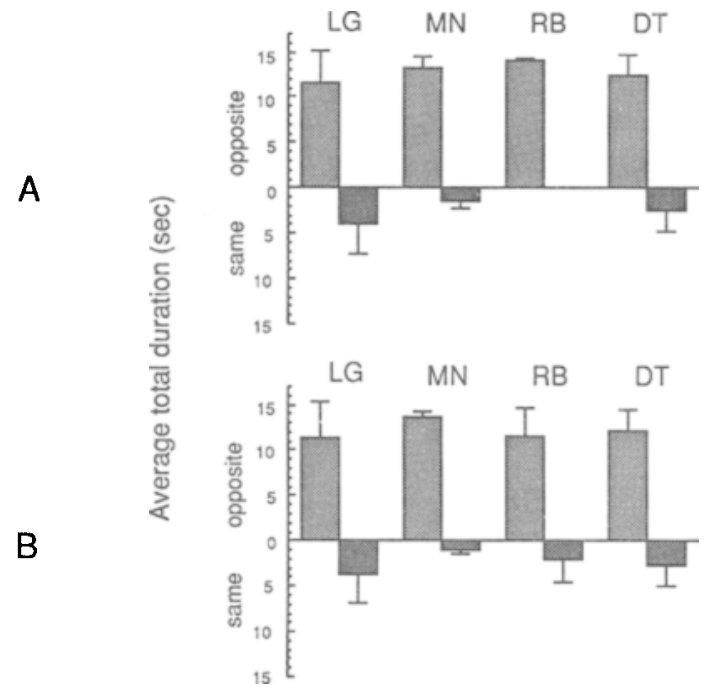

Figure 10. Average duration of perceived rotation in the opposite and in the same directions as that experienced during adaptation. (A) The 3-D adaptation figure was a cube and the 2-D test figure the rotating globe; the same pattern of results was obtained when the adaptation figure was the globe and test figure the cube. (B) The adaptation figure was the 3-D wire figure and the test figure the 2-D globe; again, the same results are obtained when globe and cube switch roles.

parable along some other stimulus dimension (e.g., their 2-D Fourier spectra).

\section{Is Stereopsis Necessary?}

As Dosher et al. (1986) have demonstrated, retinal disparity effectively disambiguates a KD figure's direction of rotation. Indeed, this is undoubtedly why stereoscopically defined 3-D figures are so effective as adaptation stimuli in our experiments. It is possible to produce unambiguous rotation of a 2-D figure, using nonstereoscopic information (Braunstein, 1977; Dosher et al., 1986; Hershberger, Stewart, \& Laughlin, 1976). Can such unambiguous 2-D figures bias the direction of rotation of an ambiguous 2-D figure? In other words, is adaptation to unambiguous rotation effective only when stereopsis is involved? To find out, we tested several different rotating 2-D figures that might be perceived as rotating in a single specific direction.

To start, we simply introduced perspective into the 2-D dot figure, a manipulation that was shown to improve observers' judgments of direction of rotation in an experiment by Braunstein (1966). Although favoring the direction of rotation specified by perspective, our observers still reported numerous reversals upon $90 \mathrm{sec}$ of viewing the perspective-enriched 2-D globe, which is also what Dosher et al. (1986) found. Hence, the 2-D perspective globe does not represent a particularly effective adapting stimulus for our purposes.

Following the lead of Petersik et al. (1984), we next tried a flat sheet of 200 dots (constructed with polar per- spective) that revolved about the midline of the sheet. Our observers, like Petersik et al.'s, tended to see this plane of dots as rotating in the direction specified by the perspective information, although reversals were sometimes experienced with this stimulus too. Despite the tendency to rotate in one direction, however, $90 \mathrm{sec}$ of adaptation to this 2-D figure failed to bias the perceived direction of rotation of the 2-D globe (Figure 11).

Next we tried a version of what Dosher et al. (1986) have called a "proximity/luminance"' cue. The adapting figure was a 2-D rotating globe whose "front-surface" dots were larger $\left(3^{\prime} \times 3^{\prime}\right)$ than the "rear-surface" dots $\left(2^{\prime} \times 2^{\prime}\right)$; because our displays were simply black and white (i.e., 2-bit gray level) we were unable to make the larger dots brighter. The additional depth information afforded by dot size did indeed bias the perceived direction of rotation of the globe; it was seen predominantly to rotate in the direction of the larger dots, which appeared to form the front surface of the globe. As an adapting stimulus, however, this unambiguous 2-D globe was ineffective, in that the subsequently viewed 2-D test globe (without proximity information) underwent spontaneous reversals in direction of rotation (see Figure 11B).

Finally, we devised a fourth unambiguous, nonstereoscopic adaptation figure. This was an "opaque sphere" generated by presentation of 100 dots all moving in a single direction; the dots appeared to be just those on the convex surface of the sphere facing the observer. Hence, as the sphere rotated, dots seemed to disappear as they passed behind what looked like the side of the sphere. Note that there is a second geometrically plausible percept associated with this stimulus-a transparent globe with dots visible only on its back surface. In fact, however, the perceived direction of rotation always coincided with the direction of motion of the dots, implying that the observers saw this object as an opaque globe. ${ }^{4}$

Adaptation to this opaque sphere was ineffective in biasing the direction of rotation of the subsequently viewed 2-D globe (Figure 11C). This result is not attributable to the reduced number of dots used to create the opaque globe, for we found that a stereo version of the opaque globe was very effective as an adaptation stimulus (Figure 11D). Depth from stereopsis thus seems to be a necessary component in the adaptation phase. Moreover, to be effective, stereoscopic adaptation must involve multiple planes of depth (e.g., the two-sheet stimulus employed earlier) or at least a disparity gradient (e.g., the stereo opaque globe); recall that a single sheet of dots at a given disparity is not an effective adaptation stimulus. Whether other depth cues such as shape-from-shading would be sufficient to produce adaptation remains to be learned.

We reasoned that if stereopsis and motion have such close connections, it should be possible for stereoscopic adaptation to influence the perception of the opaque sphere. Recall that the opaque rotating sphere was nearly always seen as convex. Since we could bias the direction of rotation of the 2-D sphere, perhaps it would be possi- 


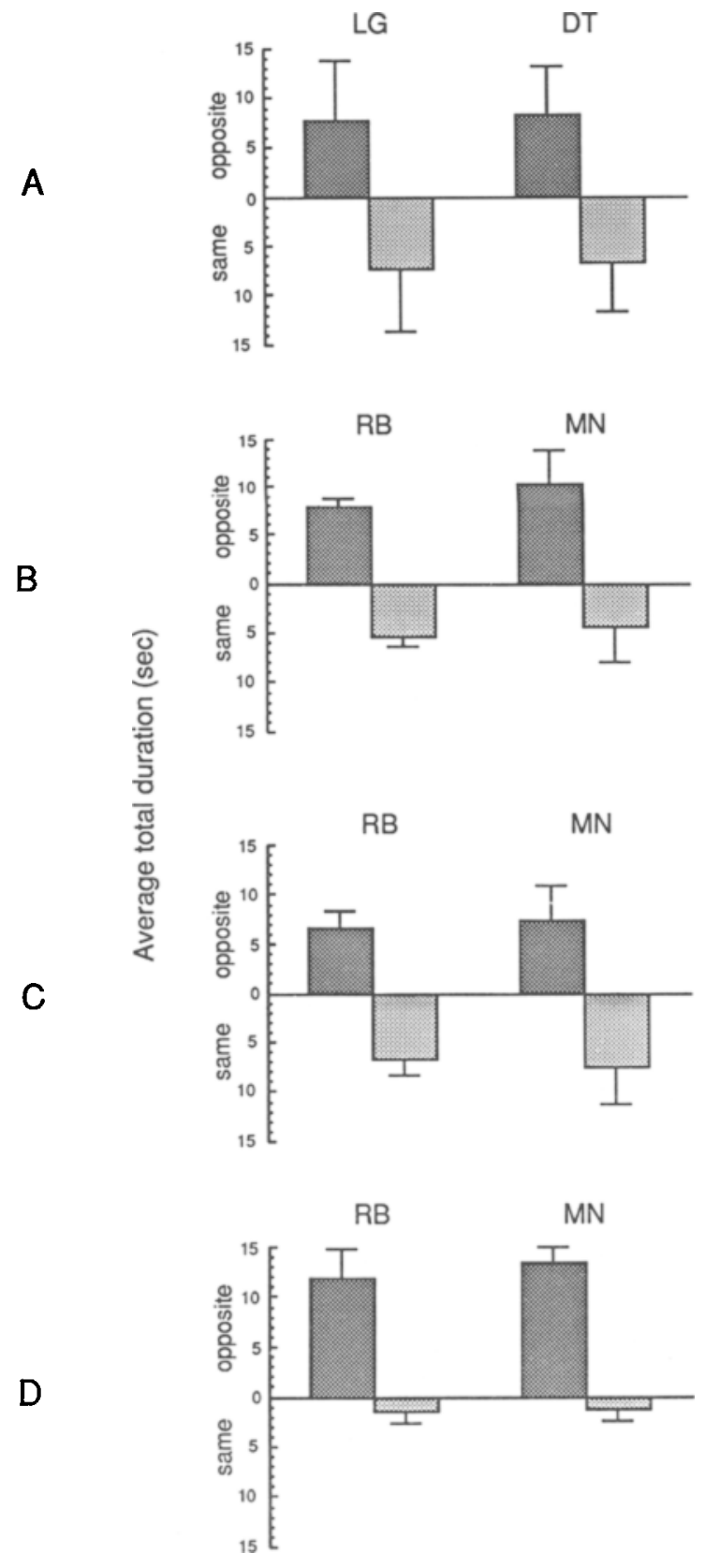

Figure 11. Effect of adapting for $90 \mathrm{sec}$ to different nonstereoscopic, rotating figures. (A) The adapting figure was a flat sheet of dots whose direction of rotation was specified by perspective. This adapting figure, though its direction of rotation was relatively stable, had no effect on the subsequent direction of rotation of the ambiguous 2-D globe. (B) The adapting figure was a 2-D globe in which the front surface dots were larger than the rear surface dots. Adaptation was ineffective. (C) The adapting figure was a 100-dot opaque globe. Adaptation was ineffective. (D) The adaptation figure was a stereoscopic version of the opaque globe. Adaptation was highly effective. ble to reverse temporarily the perceived direction of rotation of the opaque globe. This was an interesting possibility, for to alter the direction of rotation of the opaque globe would also alter the direction of curvature of the globe; the globe should appear concave, not convex. Adaptation would thus transform a perfectly reasonable stimulus into a strange figure, namely a globe having an opaque rear surface but an invisible front surface. Can 3-D adaptation create this strange percept?

To answer this question, we used a rotating 3-D sphere as the adaptation stimulus, and the opaque 2-D globe as the test stimulus. Upon adaptation to counterclockwise 3-D rotation, an opaque globe defined by dots moving left to right appeared to be concave and rotating clockwise. The reciprocal condition (clockwise 3-D rotation) yielded the converse result (a concave surface and counterclockwise 2-D rotation of dots moving right to left). These results dramatize the potent linkage between stereopsis and motion.

\section{Bias of Other KD Stimuli}

The test stimuli used so far consisted of dots, all of which moved smoothly and continuously; their motion paths, which were predetermined, coincided with the rotation of a 3-D object. In the experiments described in this section, the motion stimuli were not nearly so constrained. In recent years, in certain laboratories (Treue \& Andersen, 1990; Williams \& Phillips, 1986, 1987), novel KD stimuli have been developed that have stochastic motions: motion displays in which not all of the constituent dots have a predetermined path and velocity. Despite the stochastic nature of such displays, observers typically perceive structure and rotational motion when viewing them. It is tempting to conclude that these stochastic motion displays engage the same neural mechanisms as those stimulated by more conventional KD displays. Accordingly, we felt it would be informative to determine whether or not 3-D adaptation could bias perception of these locally random motion figures.

The first stimulus, devised by Williams and Phillips (1986), consisted of 200 moving dots whose direction of motion was limited to a subset of all possible directions. These 200 dots appeared within a circular aperture $2.5^{\circ}$ in diameter. Between cinematogram frames the dots were displaced $4^{\prime}$ according to the following two rules: From frame to frame, each dot's direction of displacement was independent of its previous displacement, and each dot's direction of displacement fell somewhere within a $120^{\circ}$ range centered on vertical (see Figure 12). This stimulus, despite its local randomness, is globally perceived as a rotating cylinder viewed through an aperture; as it rotates, the cylinder also seems to move slowly upward. The direction of rotation of this cylinder is ambiguous (i.e., it reverses between clockwise and counterclockwise over time). Can 3-D adaptation temporarily stabilize the direction of rotation of the cylinder? 


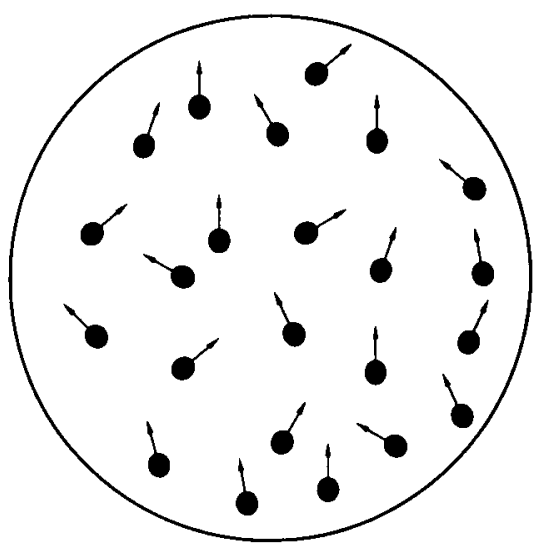

Range of possible dot movements

Figure 12. This figure summarizes possible directions of motion present in the random-dot cinematogram. From frame to frame, a dot could move in any direction $\pm 60^{\circ}$ to either side of vertical. A given dot's direction of movement varied randomly from frame to frame within this constraint. This stimulus looks like a transparent cylinder rotating either clockwise or counterclockwise, with the direction of rotation varying over time.

To answer this question, we employed the standard adaptation/test sequence. The observers viewed the 3-D adaptation sphere for $90 \mathrm{sec}$, after which they indicated the direction of rotation of the rotating stochastic cylinder during a 15-sec test period. On four trials, 3-D adaptation was clockwise, and on four other trials, it was counterclockwise. The ambiguous 2-D random motion was indeed affected by 3-D adaptation; Throughout the 15-sec test phase, the cylinder always appeared to rotate in the direction opposite that experienced during adaptation.

The second novel stimulus that we used was developed by Williams and Phillips (1987; see also Treue \& Andersen, 1990); we shall refer to it as random-velocity 1-D motion. This stimulus again comprised 200 dots randomly placed within a $2.5^{\circ}$ aperture. Between every cinematogram frame, each dot was displaced horizontally, either leftward or rightward. The size of the displacement was variable; it ranged anywhere from 1' to $6^{\prime}$ between any two frames. The size and direction (left vs. right) of each dot's displacement were independent of its displacement on previous frames. This stimulus resembled a spheroid with dots distributed throughout its volume. When displacements were horizontal, the sphere seemed to rotate about the vertical axis, with the direction of rotation varying over time. It should be stressed that this stimulus was not simply a version of dynamic visual noise of the sort studied by Tyler (1977) or by Morgan and Ward (1980); their noise stimulus required stereopsis (i.e., introduction of an interocular delay), whereas the random-velocity motion stimulus gave compelling depth and rotation even when viewing was monocular.

Observers reported on the direction of rotation of this random-velocity stimulus after a $\mathbf{9 0}$-sec adaptation phase during which they viewed the rotating 3-D sphere; both clockwise and counterclockwise adaptation were tested. This random-velocity stimulus was indeed affected by the stereoscopic adaptation figure: during the $15-\mathrm{sec}$ test phase, it always appeared to rotate in the direction opposite that experienced during adaptation.

In summary, two forms of stochastic motion are susceptible to stereoscopic adaptation. In this sense, these random-motion stimuli behave similarly to more conventional KD figures (e.g., our 2-D globe), in which rigid rotation is explicitly specified.

\section{DISCUSSION}

It is well established that a rotating 2-D figure can appear to undergo spontaneous reversals in the perceived direction of rotation; our 2-D cinematogram displays are just another example of this ubiquitous motion phenomenon. Multistable visual phenomena, including reversible rotating figures, have fascinated psychologists over the years. To explain reversible figures, workers have invoked the concept of neural fatigue, which has been variously attributed to changes in synaptic resistance (McDougall, 1906), to electrotonic spread of cortical potentials (Köhler \& Wallach, 1944), and to an autoinhibitory process (Howard, 1961). The present findings demonstrate that this putative "fatigue" process, whatever its neural basis, can be activated by prolonged viewing of an unambiguous figure, which in our experiments was a 3-D object whose direction of rotation was specified by stereoscopic information. Virsu (1975) found that stereopsis can also temporarily disambiguate perspective reversals in a static figure, the Schröder staircase.

Our results, which are summarized in the Appendix, further strengthen the case for a strong linkage between stereopsis and motion perception, a linkage already suggested by earlier psychophysical work (e.g., Howard \& Simpson, 1989; Rogers \& Graham, 1984; Smith, 1976; Wallach et al., 1963). In particular, we have found that stereopsis exerts a potent influence on the perception of structure from motion. This influence is global, in the sense that a single stereoscopic sheet of moving dots has no subsequent effect on $\mathrm{KD}$; the 3-D stimulus experienced during adaptation must consist of a complexdisparity field of moving dots. Moreover, the influence of stereopsis on motion generalizes across figural shapes (e.g., a globe adapts a cube). At the same time, the influence is specific to the retinal area stimulated by the adaptation stimulus, implying that the underlying neural processes operate on motion fields defined in retinal coordinates.

Chang (1990) has recently investigated the interaction between motion defined by luminance information and depth defined from random-element stereograms. In a sense, her work complements ours. She studied how motion signals portrayed by luminance information could bias the perception of direction of motion of cyclopean contours defined by disparity. In general, she found that a 
cyclopean pattern portrayed in a random-element stereogram appeared to move smoothly only when the constituent luminance dots also moved in a direction consistent with the cyclopean movement. Our results, as well as those of Dosher et al. (1986), show the converse; luminance dots defining a 2-D figure appear to move smoothly and unambiguously if stereopsis defines the location in depth of those dots.

Petersik et al. (1984) have also studied reversals in perceived direction of rotation of ambiguous figures. Using monocular perspective cues to disambiguate motion, they found that adaptation to unambiguous rotation temporarily caused an ambiguous-motion stimulus to rotate in the direction opposite that experienced during adaptation. This, of course, represents a nonstereoscopic version of the effect that we studied. Similarly, Rogers and Graham (1984) were able to bias the perception of ambiguous motion by prior adaptation to unambiguous structure from motion. We were unsuccessful, however, in biasing 2-D motion using a nonstereoscopic adaptation stimulus (i.e., the opaque globe, the proximity/size globe, the perspective plane). One could argue that the earlier experiments of Rogers and Graham (1984) and Petersik et al. (1984) involve a "depth"' aftereffect, whereas our work involves a "motion" aftereffect. This argument is based on an inappropriate characterization of our findings, though. The phenomenon explored in our work is, in fact, a depth aftereffect, one that is contingent on motion. During adaptation, the observer views motion in a given direction at a given disparity plane; following adaptation, figure elements moving in that direction appear to occupy a different depth plane. It is the plane of depth that is "misperceived," not the direction of motion. Moreover, the plane of depth of stationary dots is not misperceived (although the dots may appear to move); the depth aftereffect produced by motion in a given direction at a given disparity plane is contingent on the presence of motion in the same direction during the postadaptation test phase. Thus while agreeing with Petersik et al. (1984) and with Rogers and Graham (1984) that adaptation to perceived motion in depth affects an ambiguous $\mathrm{KD}$ figure, we are left wondering about the sufficient conditions for defining depth in the adaptation stimulus.

With respect to the physiological basis of the stereopsis/motion interaction studied here, it has become fashionable to think of different aspects of the visual scene as being processing in separate pathways. The latest version of this idea has been championed by Livingstone and Hubel $(1987,1988)$, the emphasis being on the so-called magnocellular and parvocellular pathways (named according to the layer of the lateral geniculate nucleus from which the projections originate). According to this scheme, the magnocellular pathway processes information underlying motion perception and stereopsis while the parvocellular pathway mediates color and form perception. This idea has generated considerable debate (see, e.g., Shapley, 1990; Schiller, Logothetis, \& Charles, 1990). The present data have no critical bearing on this question of parallel pathways other than to provide prima facie evidence that motion and stereopsis share a common neural basis, or at least arise from processes that strongly interact. It has not escaped our attention that neurons comprising the magnocellular pathway are particularly responsive to motion and, at higher stages, are selective for retinal disparity (DeYoe \& Van Essen, 1988). At still higher stages in this pathway are visual centers (the middle superior temporal cortical area) whose neurons are responsive to rotation and to expansion (Saito et al., 1986; Tanaka et al., 1986). In general, neurons in this occipitotemporal processing stream possess the kinds of receptive field properties one would expect to find in neural mechanisms underlying kinetic depth perception.

Inspired by these suggestive physiological data, we (Nawrot \& Blake, 1990) have developed a neural model of $\mathrm{KD}$ and its interaction with stereopsis. ${ }^{5}$ The model posits binocularly activated units selective for both disparity and direction of motion. Each unit is directly activated by a given direction of motion imaged at a particular retinal disparity. The activity of these binocular units is also determined by connections from other binocular units preferring the same direction of motion; for units whose preferred disparities are similar, this influence is facilitatory, and for units whose preferred disparities are quite different, this influence is inhibitory. At a given disparity plane, units preferring opposite directions of motion are mutually inhibitory. In response to a 2-D KD stimulus of the sort used in our experiments (i.e., a rotating globe), activity in the network quickly segregates into disparity planes other than the zero-disparity plane defined by the physical stimulus. This segregated pattern of activity fluctuates with a time course mimicking the reversals in perceived direction of rotation of a 2-D KD stimulus. The addition of disparity information to the stimulus activates disparity planes appropriate for the stimulus, and, moreover, this pattern of activity is stable. Immediately following removal of stimulus disparity information, the network activity pattern switches to one signaling rotation in the opposite direction and remains in this activity pattern for an unusually long period. This behavior, in other words, mimics the psychophysical observations described in this paper.

\section{REFERENCES}

ANSTIS, S. M., \& IARRIS, J. P. (1974). Movement aftereffects contingent on binocular disparity. Perception, 3, 153-168.

BLAKEMORE, C., Julesz, B. (1971). Stereoscopic depth aftereffect produced without monocular cues. Science, 171, 286-288.

Borsellino, A., De Marco, A., Allazetta, A., Rinesi, S., \& BarTOLIN, B. (1972). Reversal time distribution in the perception of visual ambiguous stimuli. Kybernetik, 10, 139-144.

Bradshaw, M. F., Frisby, J. P., Mayhew, J. E. W. (1987). The recovery of structure from motion: No evidence for a special link with the convergent disparity mechanism. Perception, 16, 351-357.

Braunstein, M. L. (1962). The perception of depth through motion. Psychological Bulletin, 59, 422-433.

Braunstein, M. L. (1966). Sensitivity of the observer to transformations of the visual field. Joumal of Experimental Psychology, 72, 683-689. 
Braunstein, M. L. (1977). Perceived direction of rotation of simulated three-dimensional patterns. Perception \& Psychophysics, 21, 553-557.

Braunstein, M. L., \& Andersen, G. J. (1984). A counterexample to the rigidity assumption in the visual perception of structure from motion. Perception, 13, 213-217.

BurR, D. C., \& Ross, J. (1979). How does binocular delay give information about depth? Vision Research, 19, 523-532.

Chang, J. J. (1990). New phenomena linking depth and luminance in stereoscopic motion. Vision Research, 30, 137-147.

De Marco, A., Penengo, P., Trabucco, A., Borsellino, A., Carlini, F., Riani, M., \& Tuccio, M. T. (1977). Stochastic models and fluctuations in reversal time of ambiguous figures. Perception, 6, 645-656.

DeYoe, E. A., \&AN Essen, D. C. (1988). Concurrent processing streams in monkey visual cortex. Trends in Neuroscience, 11, 219-226.

Dosher, B. A., Sperling, G., Wurst, S. A. (1986). Tradeoffs between stereopsis and proximity luminance covariance as determinants of perceived 3D structure. Vision Research, 26, 973-990.

Eвy, D. W., Loomis, J. M., Solomon, E. M. (1989). Perceptual linkage of multiple objects rotating in depth. Perception, 18, 427-444.

FisiCHELLI, V. R. (1947). Reversible perspective in Lissajous' figures: Some theoretical considerations. American Joumal of Psychology, 60 240-249.

Fox, R., Herrmann, J. (1967). Stochastic properties of binocular rivalry alternations. Perception \& Psychophysics, 2, 432-436.

Fox, R., Patterson, R., Lehmxuhle, S. (1982). Effect of depth position on the motion aftereffect. Investigative Ophthalmology \& Visual Science, 22, 144.

Helmholtz, H. (1962). Treatise on physiological optics (3rd ed.; J. P. C. Southall, Trans. \& Ed.). New York: Dover. (Original work published 1909)

Hershberger, W. A., Stewart, M. R., \& Laughlin, N. K. (1976). Conflicting motion perspective simulating simultaneous clockwise and counterclockwise rotation in depth. Journal of Experimental Psychology: Human Perception \& Performance, 2, 174-178.

HowARD, I. P. (1961). An investigation of a satiation process in the reversible perspective of revolving skeletal shapes. Quarterly Journal of Experimental Psychology, 9, 19-33.

Howard, I. P., Simpson, W. A. (1989). Human optokinetic nystagmus is linked to the stereoscopic system. Experimental Brain Research, 78, 309-314

Julesz, B. (1971). Foundations of cyclopean perception. Chicago: University of Chicago Press.

KöHLER, W., \& WALACH, H. (1944). Figural after-effects: An investigation of visual processes. Proceedings of the American Philosophical Society, 88, 269-357.

LAPPIN, J. S., \& FUQUA, M. A. (1983). Accurate visual measurement of three-dimensional moving patterns. Science, 221, 480-482.

Livingstone, M. S., \& Hubel, D. H. (1987). Psychophysical evidence for separate channels for the perception of form, color, movement, and depth. Joumal of Neuroscience, 7, 3416-3468.

Livingstone, M. S., \& HubEL, D. H. (1988). Segregation of form, color, movement, and depth: Anatomy, physiology, and perception. Science, 240, 740-749.

LU, C., \& FeNDER, D. H. (1972). The interaction of color and luminance in stereoscopic vision. Investigative Ophthalmology, 11, 482-490.

MCDougall, W. (1906). The physiological factors in the attention process. Mind, 15, 329-359.

MORGAN, M. J., \& WARD, R. (1980). Interocular delay produces depth in subjectively moving noise patterns. Quarterly Joumal of Experimental Psychology, 32, 387-395.

NAWROT, M., \&LAKE, R. (1989). Neural integration of information specifying structure from stereopsis and motion. Science, 244, 716-718.

NAWROT, M., BLAKE, R. (1990). A network model of interactions between motion and stereopsis in the specification of structure. Investigative Ophthalmology \& Visual Science, 31, 529.

NAWROT, M., \& BLAKE, R. (in press). A neural network model of kinetic depth. Visual Neuroscience.
Petersik, J. T., Shepard, A., \& Malsch, R. (1984). A threedimensional motion aftereffect produced by prolonged adaptation to a rotation simulation. Perception, 13, 489-497.

RAMACHANDRAN, V. S., \& GREgoRy, R. L. (1978). Does colour provide an input to human motion perception? Nature, 275, 55-56.

RICHARDS, W. (1985). Structure from stereo and motion. Journal of the Optical Society of America A, 2, 343-349.

Richards, W., LeIBERMAN, H. R. (1985). Correlation between stereo ability and the recovery of structure from motion. American Journal of Optometry \& Physiological Optics, 62, 111-118.

Rogers, B. J., \&RAHAM, M. E. (1982). Similarities between motion parallax and stereopsis in human depth perception. Vision Research, 22, 261-270.

Rogers, B. J., \& Graham, M. E. (1983). Anisotropies in the perception of three-dimensional surfaces. Science, 221, 1409-1411.

Rogers, B. J., \& GrahAM, M. E. (1984). Aftereffects from motion parallax and stereoscopic depth: similarities and interactions. In L. Spillman \& B. R. Wooten (Eds.), Sensory experience, adaptation, and perception: Festschrift for Ivo Kohler (pp. 603-619). Hillsdale, NJ: Erlbaum.

Saito, H., Yukie, M., Tanaka, K., Hikosaka, K., Fukada, Y., \& IWAI, E. (1986). Integration of direction signals of image motion in the superior temporal sulcus on the macaque monkey. Journal of Neuroscience, 6, 145-157.

Shapley, R. (1990). Visual sensitivity and parallel retinocortical channels. Annual Review of Psychology, 41, 635-658.

SCHILler, P. H., Logothetis, N. K., \& Charles, E. R. (1990). Functions of the colour-opponent and broad-band channels of the visual system. Nature, 343, 68-70.

SMITH, R. A., JR. (1976). The motion/disparity aftereffect: A preliminary study. Vision Research, 16, 1507-1509.

Speruing, G., Landy, M. S., Dosher, B. A., \& PerkINS, M. E. (1989). Kinetic depth effect and identification of shape. Joumal of Experimental Psychology: Human Perception \& Performance, 15, 826-840.

Tanaka, K., Hikosaka, K., Saito, H., Yukie, M., Fukada, Y., \& IWAI, E. (1986). Analysis of local and wide-field movements in the superior temporal visual areas of the macaque monkey. Journal of Neuroscience, 6, 134-144.

ToDD, J. T. (1984). The perception of three-dimensional structure from rigid and nonrigid motion. Perception \& Psychophysics, 36, 97-103.

ToDD, J. T. (1985). Perception of structure from motion: Is projective correspondence of moving elements a necessary condition? Journal of Experimental Psychology: Human Perception \& Performance, 11, 689-710.

Toppino, T. C., LoNG, G. M. (1987). Selective adaptation with reversible figures: Don't change that channel. Perception \& Psychophysics, 42, $37-48$.

Treue, S., ANDERSEN, R. A. (1990). 3-D Structure from motion: Rigidity and surface interpolation. Investigative Ophthalmology \& Visual Science, 31, 172.

TYLER, C. W. (1977). Stereomovement from interocular delay in dynamic visual noise: A random spatial disparity hypothesis. American Journal of Optometry \& Physiological Optics, 54, 374-386.

VIRSU, V. (1975). Determination of perspective reversals. Nature, 257, 786-787.

WALLACH, H., \& KARSH, E. B. (1963). The modification of stereoscopic depth-perception and the kinetic-depth effect. American Journal of Psychology, 75, 429-435.

Wallach, H., MOORE, M. E., \& DAvidson, L. (1963). Modification of stereoscopic depth perception. American Journal of Psychology, 75, 191-204.

Wallach, H., O'ConNell, D. N. (1953). The kinetic depth effect. Joumal of Experimental Psychology, 45, 205-217.

Watson, A. B., AhUmada, A. J., JR. (1985). Model of human visual motion sensing. Journal of the Optical Society of America A, 2 , 322-341.

WheATSTONE, C. (1838). Contributions to the physiology of vision: I. On some remarkable, and hitherto unobserved, phenomena of binocular vision. Philosophical Transactions of the Royal Society, 128, 371-394. 
Willisms, D., \& Phillips, G. (1986), Structure from motion in a stochastic display. Journal of the Optical Society of America A, 3, 12. Willuams, D., PHILuIs, G. (1987). Rigid 3-D percept from stochastic 1-D motion. Joumal of the Optical Society of America A, 4, 35.

\section{NOTES}

1. Unambiguous depth from motion in the Rogers and Graham (1984) motion displays can be produced either by moving the head or by moving the display concomitantly with translation of the motion elements. In the rotating $\mathrm{KD}$ displays used in the present experiments (created by parallel projection of motion elements), velocity of motion elements specifies relative distances within the object but not distance from the observer to the object. For this reason, depth is reversible in the $\mathrm{KD}$ display.

2. We lowered the luminance of the left-eye display by $0.4 \log$ units to eliminate a residual stereoscopic cue. Specifically, this slight luminance mismatch counteracted the brief interocular time disparity caused by sequential presentation of frames on the two monitors. This temporal disparity, in the absence of luminance compensation, produced a stereoscopic cue in the absence of spatial disparity (Burr \& Ross, 1979). With luminance compensation, the 2-D globe contained no disparity information and therefore appeared to be bistable.

3. During reversals, the figure does not seem to cease rotating and then resume rotation in the opposite direction. Instead, the object appears to twist within itself in a nonrigid fashion, such that the direction of the rearmost surface becomes the direction of the frontmost surface and vice versa. This peculiar percept has no obvious "real world" analogue; it is most likely an illusory effect caused by the transparency of the environmentally uncommon and ambiguous stimulus properties of the KDE.

4. The authors, who have had considerable experience viewing this opaque 2-D globe, occasionally see it as concave rather than convex. The role of familiarity in generating this percept is quite interesting and deserves examination.

5. The model was constructed to deal explicitly with the perception of depth in dynamic displays devoid of retinal disparity information. It does not deal with the perception of shape/structure from such displays.

APPENDIX

Summary of Experimental Results

Question $\quad$ Adaptation Stimulus

Temporal properties

Stereo Adaptation

Recovery from stereo

adaptation

Retinal specificity

Adaptation to sheets in
stereo depth

Monocular versus

binocular viewing of test

figure

Axis of rotation

Stationary 3-D test

figure

Shape specificity

Perspective adaptation

Perspective sheet

Changing size depth cue

Opaque globe
3-D flat sheets

3-D globe

3-D globe, horizontal axis

3-D globe

3-D cube, 3-D globe

2-D globe

2-D rotating plane with perspective

2-D globe with proximity cue

2-D opaque globe, 3-D opaque globe

Test
2-D globe
2-D globe
2-D globe
2-D globe

2-D globe

2-D globe

2-D globe, vertical axis

Stationary 3-D globe

2-D globe, 2-D cube, 2-D wire figure

2-D globe with perspective

2-D globe

2-D globe

2-D globe
Result

Successive durations are stochastically independent

Adaptation temporarily disambiguates perceived direction of rotation

Recovery from adaptation occurs within $30 \mathrm{sec}$

Adaptation is specific to retinal location of adaptation figure

Two 3-D sheets needed to bias perceived direction of rotation

Bias found with both binocular and monocular viewing of test figure

Adaptation ineffective

No rotational aftereffect

Adaptation and test figures need not be the same shape for adaptation to be effective

Adaptation does not disambiguate perceived direction of rotation

Adaptation does not disambiguate perceived direction of rotation

Adaptation does not disambiguate perceived direction of rotation

Adaptation does not disambiguate perceived direction of rotation 


\section{NAWROT AND BLAKE}

Question

Does stereo affect 2-D

random motion

Does stereo affect 1-D random motion
Adaptation Stimulus

3-D globe

3-D globe
Test Stimulus

2-D random motion

1-D random motion
Result

Adaptation temporarily disambiguates perceived direction of rotation

Adaptation temporarily disambiguates perceived direction of rotation

(Manuscript received June 8, 1990;

revision accepted for publication October 9, 1990.) 